\title{
Volumetric-Modulated Arc Radiotherapy with Daily Image-Guidance Carries Better Toxicity Profile for Higher Risk Prostate Cancer
}

\author{
Ahmed I Ghanem ${ }^{1,2}$, Amr A Elsaid ${ }^{2}$, Mohamed A Elshaikh¹, Gehan A Khedr ${ }^{2 *}$
}

\begin{abstract}
Purpose: To compare radiotherapy-induced toxicity for localized prostate-cancer (PCa) treated with versus without daily image-guidance. Patients and Methods: We identified consecutive intermediate and high-risk localized PCa patients treated with definitive radiotherapy using intensity-modulated radiotherapy (IMRT) with variable duration of androgen-deprivation therapy (ADT) within 2015-2016 (Arm-A) and 2005-2007 (Arm-B). Arm-A cases received daily online imaging guidance (IGRT) using cone-beam computed tomography (CBCT) unlike Arm-B candidates with no daily IGRT. After reporting demographic, clinico-pathological features and treatment details, we compared acute (within 3 months post-therapy) and late RT-induced toxicities between study groups graded by RTOG/CTCAE criteria. Uni/multivariate analyses (UVA/MVA) were performed to identify independent predictors for RT-related side-effects. Results: We were able to identify 257 cases who met our inclusion criteria. Overall, median age was 73 years (48-85), 67\% had intermediate-risk and 47\% received ADT. Arm-A included 72 patients who received IMRT delivered using volumetric-modulated arc therapy (VMAT), whereas, Arm-B was formed of 185 cases who utilized step-and-shoot static IMRT. Clinico-pathological features and treatment details were non-different across study arms except that Arm-A had more Grade Group 3, higher median total dose (79.2 vs. 74 Gy) and more pelvic lymph-nodes RT $(\mathrm{P}<0.05)$. Although acute toxicity was similar across groups, Arm-B encountered higher late toxicity score, more intense late genitourinary side-effects $(\mathrm{P}=0.008)$, with non-different late lower-gastrointestinal toxicities. On MVA, lack of daily $\mathrm{CBCT}$, African-American race and higher comorbidities were independently predictive for late toxicities. Conclusion: IMRT with daily CBCT permitted safe delivery of dose-escalated IMRT with improved toxicity profile for higher-risk prostate cancer.
\end{abstract}

Keywords: Acute and late toxicity- image-guided radiotherapy- intermediate and high-risk

Asian Pac J Cancer Prev, 22 (1), 61-68

\section{Introduction}

Prostate cancer $(\mathrm{PCa})$ is the most common male malignancy and the second cause of cancer death in the United States (US) with expected 248,530 new cases and 34,130 deaths in 2021 (Siegel et al., 2021). Following the 2012 update of the US Preventive Task Force against routine prostate specific antigen (PSA) screening (Moyer, 2012), there has been an increase in the proportion of higher risk localized cases especially in those $>75$ years at diagnosis (Hu et al., 2016).

According to the National Cancer Comprehensive Network (NCCN) guidelines the majority of PCa patients of intermediate and high-risk with long life expectancy will end up receiving either radical prostatectomy (RP), or definitive radiotherapy (RT) with or without androgen deprivation therapy (ADT) (National Comprehensive Cancer Network, 2019). Men with higher risk PCa receiving definitive $\mathrm{RT}$ tend to be relatively older with increased comorbidity burden compared to RP candidates, as reported in multiple population-based studies (Buglione et al., 2019; Daskivich et al., 2013).

Definitive RT modalities, techniques and doses represent an evolving field for advances and updates aiming at delivering a curative dose to the prostate with the least possible side-effects that result from irradiating nearby organs at risk (OAR) (Zaorsky et al., 2013; Zaorsky et al., 2017). Intensity modulated radiotherapy (IMRT) has been established as a standard of care for external beam RT for PCa based upon various studies that have depicted a lower rate of acute and late RT-induced toxicities compared to 3D-conformal RT (3D-CRT). Improved toxicity profile was more pronounced for the lower gastrointestinal (GI) more than genitourinary (GU) side-effects (Zelefsky et al., 2008; Michalski et al., 2013; Wortel et al, 2016). Nonetheless, none of these were 
randomized to compare both techniques. Nowadays, delivery of IMRT is achieved more commonly by either multiple static fields (step-and-shoot) or the evolving Volumetric Modulated Arc Therapy (VMAT) with rotating arcs (Zaorsky et al., 2017).

With the higher level of dose conformality achieved with modern RT modalities, higher doses had been tested for higher risk PCa. In fact, randomized trials comparing escalated doses (76-80 Gy) vs. lower doses (68-70 Gy) showed consistent significant improvement in biochemical control with some showing less clinical recurrences as well (Kuban et al, 2007; Heemsbergen et al, 2014). However, most patients of these studies received 3D-CRT and suffered from more side-effects in the high dose arms; particularly GI toxicities. The Radiation-Therapy Oncology Group (RTOG)-0126 trial was the only randomized trial that allowed IMRT utilization after the protocol amendment albeit 3D-CRT was received by the majority (66.8\%) (Michalski et al., 2013).

With the establishment of dose-escalation and the significantly improved dosimetry and toxicity profile with IMRT, many tools have been tested to account for setup errors and inter-fractional displacement of the prostate to avoid coverage misses which may render IMRT not beneficial or even harmful. This contributed to the development of image-guided RT (IGRT) concept which involves imaging to verify the position of the prostate and surrounding OAR with the possibility of shifts to ensure proper dose delivery. Also, IGRT aids minimizing planning target volume (PTV) margins, which also improves OAR dosimetry boosting the therapeutic ratio (Zarosky et al., 2017). IGRT can be applied using radio-opaque fiducials that are aligned to PTV either using 2-D kV imaging, electronic portal imaging device (EPID), or more recently using cone-beam CT (CBCT) mounted in the radiotherapy delivery machine. $\mathrm{CBCT}$ can be used based on soft tissue visualization avoiding the invasive procedure for fiducials insertion (Mayyas et al., 2013; Hammoud et al., 2008). Besides, 3D-ultrasound (3D-US) can be used for IGRT in addition to the electromagnetic transponders which can provide real-time prostate position data (Scarbrough et al., 2006; Willoughby et al., 2006).

Therefore, the aim of this study is to examine the impact of contemporary IMRT with daily IGRT on acute and late treatment-related side-effects for intermediate and high-risk PCa patients treated definitively with dose-escalated RT with or without ADT, compared to a retrospective arm treated without daily IGRT in a single institution setting.

\section{Materials and Methods}

\section{Patients and methods}

After obtaining institutional review board approval, we identified consecutive patients with NCCN defined intermediate and high-risk localized PCa patients treated with definitive RT delivered by IMRT with daily IGRT using CBCT between 1/2015-1/2016; Arm-A (National Comprehensive Cancer Network, 2019). As a control we queried our $\mathrm{PCa}$ database for a similar group of patients
(Arm-B) who received IMRT without daily IGRT within 2005-2007 at the same institution. We selected this time frame as it was the latest interval before the establishment of daily CBCT as a standard of care. Arm-B cases were positioned daily relying solely on skin tattoos with inconsistent weekly/biweekly image verification for setup using on-table 3D-US or two-dimensional $\mathrm{kV}$ portal imaging.

Treatment in both groups was prescribed to the entire prostate and the seminal vesicles $+/$ - pelvic lymph-nodes (LN) according to the calculated risk and provider's discretion and, was delivered with conventional fractionation (1.8-2.0 Gy/fraction). All patients were simulated and treated with comfortably full bladder and empty rectum without using a routine enema, and also without fiducial markers insertion. PTV was formed of the prostate and proximal seminal vesicles (CTV) in addition to $1 \mathrm{~cm}$ all around except posteriorly $(5 \mathrm{~mm})$. IMRT plans were optimized to ensure adequate coverage for PTV keeping organs at risk within the predetermined constraints as possible. Dose-constraints for the rectum and urinary bladder are based on percentage of volume $(\mathrm{V})$ receiving $50,65,70$ or 75 Gy according to QUANTEC (ex: V50 and V70 for rectum and V-65 and V-70 for bladder) (Bentzen et al., 2010). ADT using gonadotrophic-releasing hormone agonist/antagonist \pm initial antiandrogen phase was administered within the process of shared-decision between the patient and consultant taking into consideration risk factors, baseline comorbidities and patient preferences.

Acute and late RT-related toxicities were graded by radiation oncologists based on RTOG and Common Terminology Criteria for Adverse-Events version 4 (2010) (RTOG/CTCAE) considering the worst grade observed along follow up time focusing mainly on lower GI, GU toxicities and erectile dysfunction (Cox et al., 1995). Acute toxicity was prospectively recorded during weekly visits throughout RT course using mainly a comprehensive checklist in addition to phone calls and any post-RT visits up to 3 months. On the other hand, late side-effects were tracked $>3$ months post-RT through radiation-oncology and urology visits until last follow-up per patients' electronic records.

After reporting demographics, baseline comorbidities using Charlosn Comorbidity Index (CCI), prognostic factors and treatment details, we compared the distribution of RT toxicities between study groups (Charlson et al., 1987). In addition to individual toxicities we reported the occurrence of any grade- 2 or more (G-2+) side-effects, we calculated a total toxicity score including maximal grade of common toxicities per patient.

We used Chi-Squared or Fisher-Exact test for categorical and Kruskal-Wallis test for continuous data. Univariate analysis followed by multivariate analyses (MVA) with Cox regression analysis including only factors with P-value $<0.1$ in addition to crucial risk factors were performed to identify independent predictors of toxicity endpoints whenever feasible. A two-sided P-value of $<0.05$ was considered statistically significant. All statistical analyses were performed using Statistical Analysis Software, version 9.4 (SAS Institute, Inc. Cary, NC, USA). 


\section{Results}

We identified 257 patients who met our inclusion criteria after excluding those who received hypo-fractionated course, those treated with a brachytherapy boost, in addition to cases with inadequate follow-up. Arm-A encompassed 72 cases (28\%) while Arm-B patients formed the rest $(n=185 ; 72 \%)$. For the entire cohort, median age was 73 years (48-85), AfricanAmericans formed 53.3\% and CCI of $2+$ was detected in $49 \%$. With a median PSA of $8.8 \mathrm{ng} / \mathrm{dl}$ and T2b-T3b of $15.1 \%$ and a median total Gleason score of 7 (6-10); NCCN intermediate-risk constituted $66.9 \%(n=172)$ of the study candidates.

Arm-A received IMRT delivered via VMAT with a median of 2 arcs and 1 arc in phase- 1 and II respectively with a median beam time of 2 minutes. Arm-B cases
IMRT was underwent with step-and-shoot technique with a median of 9 fields/phase (6-11) delivered in a median of 5.5 minutes. ADT was utilized by $46.7 \%$ of all patients with a mean total duration of 8 months (2-40).

Study arms were well-balanced for most of the baseline characteristics as well as treatment details except that Arm-A included less Grade Group 4/5 and more Grade Group 3 cases $(\mathrm{P}=0.014)$ as demonstrated in Table.1. Besides, Arm-A received significantly higher median total dose (79.2 vs $74 \mathrm{~Gy})$, were treated more with $1.8 \mathrm{~Gy} /$ fraction and more commonly had pelvic lymph-nodes irradiated; $\mathrm{P}<0.001$ for all. A trend for getting close (borderline) to or beyond predetermined constraints for both bladder $(\mathrm{P}=0.07)$ and rectum $(\mathrm{P}=0.08)$ was detected higher in Arm-A as depicted in Table. 2 which may be due to higher delivered total dose.

A great proportion of the study cohort suffered from

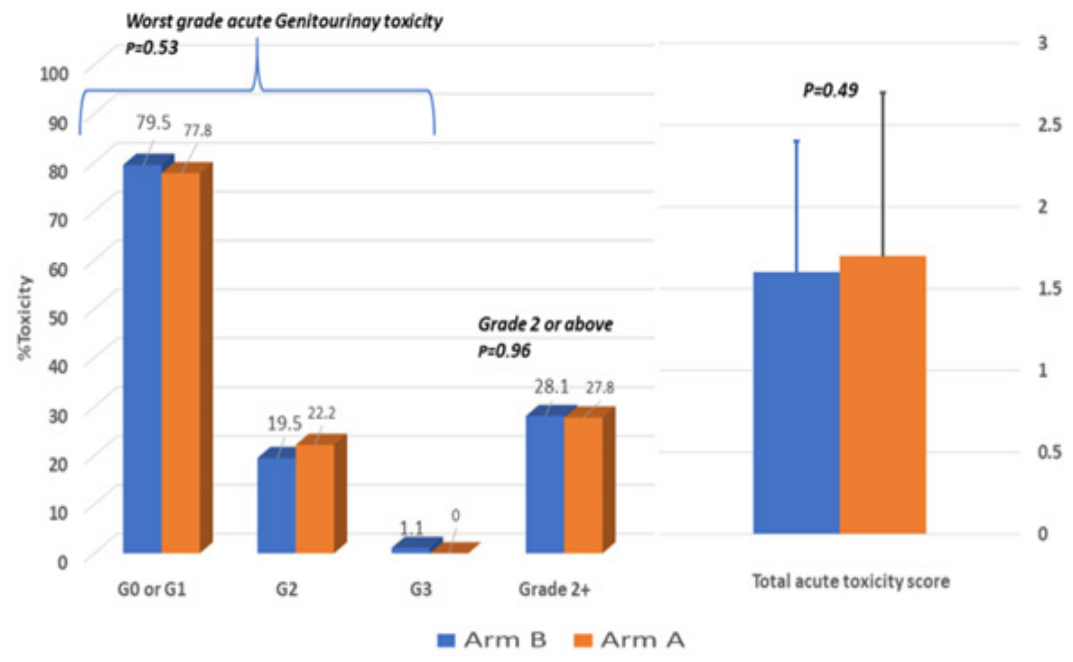

Figure 1. Acute Radiotherapy-Induced Toxicity between Arm-A (VMAT-IMRT with daily CBCT; N=72) and Arm-B (static IMRT without daily CBCT; $\mathrm{N}=185$ ) measured during weekly visits during radiotherapy up to three months post-therapy with worst grade recorded per RTOG/CTCAE.

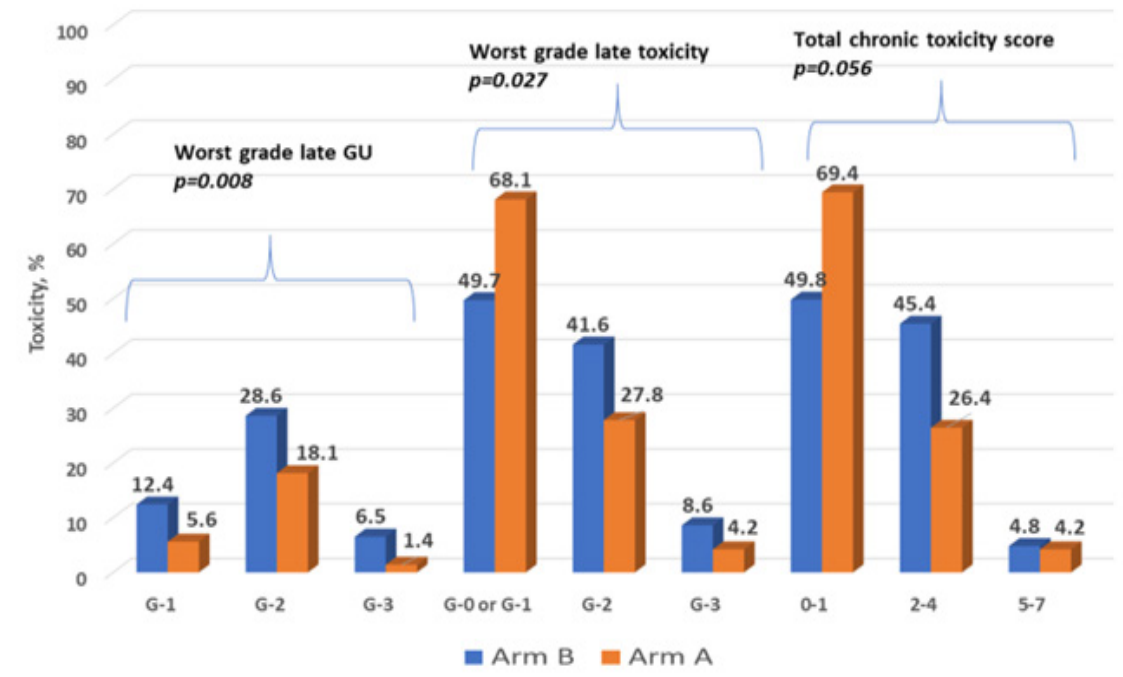

Figure 2. Late Radiotherapy-Induced Side-Effects between Arm-A (VMAT-IMRT with daily CBCT; N=72) and Arm$\mathrm{B}$ (static IMRT without daily CBCT; $\mathrm{N}=185$ ) measured during radiotherapy and urology surveillance visits after three months post-therapy up to latest follow-up with worst grade recorded per RTOG/CTCAE. 
Table 1. Baseline Demographic, Clinic-Pathological and Prognostic Characteristics for Prostate-Cancer Patients Receiving IMRT with (Arm-A) or without Daily Image-Guidance (Arm-B)

\begin{tabular}{|c|c|c|c|}
\hline Characteristic & $\begin{array}{c}\text { Arm-A } \\
(\mathrm{N}=72 ; 28 \%)\end{array}$ & $\begin{array}{c}\text { Arm-B } \\
(\mathrm{N}=185 ; 72 \%)\end{array}$ & P-value \\
\hline Median follow up (years) & 3.7 (range 3.3-4.1) & 11.2 (range $7-14.6$ ) & $<0.001$ \\
\hline Median age (years) & 71 (range 50-85) & 73 (range 48-85) & 0.7 \\
\hline African-American race & $34(47.2 \%)$ & $103(55.7 \%)$ & 0.22 \\
\hline Ever smoker (current/ex-smoker) & $45(62.5 \%)$ & $120(64.9 \%)$ & 0.72 \\
\hline Alcohol use & & & 0.41 \\
\hline Social & $17(23.6 \%)$ & $58(31.4 \%)$ & \\
\hline Frequent/abuse & $11(15.3 \%)$ & $30(16.2 \%)$ & \\
\hline Median baseline CCI score & $2(0-9)$ & $1(0-7)$ & 0.84 \\
\hline Median AUA/IPSS score at diagnosis & $5.0(2.0-9.0)$ & $5.0(2.0-10.0)$ & 0.14 \\
\hline PSA level at diagnosis (ng/dl) & $11.1 \pm 8.9$ & $12.2 \pm 10.3$ & 0.39 \\
\hline Grade Group & & & 0.014 \\
\hline $1(3+3)$ or $2(3+4)$ & $39(54.2 \%)$ & $109(58.9 \%)$ & \\
\hline $3(4+3)$ & $18(25 \%)$ & $29(15.7 \%)$ & \\
\hline $4(4+4,3+5 \& 5+3)$ or $5(4+5,5+4 \& 5+5)$ & $15(20.8 \%)$ & $47(25.4 \%)$ & \\
\hline Median number of positive cores & 4.0 (range $1.0-13.0$ ) & 5.0 (range $1.0-18.0$ ) & 0.14 \\
\hline Mean percentage of positive cores & $42.0 \pm 25.5$ & $66.6 \pm 26.4$ & $<0.001$ \\
\hline Clinical T-stage & & & 0.48 \\
\hline $\mathrm{T} 1 \mathrm{~b}-\mathrm{T} 2 \mathrm{c}$ & $69(95.8 \%)$ & $182(98.4 \%)$ & \\
\hline T3a-b & $3(4.2 \%)$ & $3(1.6 \%)$ & \\
\hline NCCN risk group & & & 0.41 \\
\hline Intermediate & $51(70.8 \%)$ & $121(65.4 \%)$ & \\
\hline High & $21(29.2 \%)$ & $64(34.6 \%)$ & \\
\hline Median UCSF-CAPRA score at diagnosis & $5.0(2.0-9.0)$ & $5.0(2.0-10.0)$ & 0.14 \\
\hline
\end{tabular}

IMRT, Intensity-Modulated Radiation Treatment; CCI, Charlson Comorbidity-Index; IPSS/AUA, International Prostate Symptom Score / American Urological Association; PSA, Prostatic Specific Antigen; NCCN, National Comprehensive Cancer Network; UCSF-CAPRA, University of California, San Francisco Cancer of the Prostate Risk Assessment

acute RT-induced side-effects with 54 cases developing G-2 GU toxicities $(21 \%)$ and only 2 cases $(0.7 \%)$ having G-3, whereas, only 21 patients had G-2 acute GI toxicity (8.2\%) with no G-3 detected. Albeit, acute toxicity did not differ significantly across treatment arms as shown in Figure. 1 including the total acute toxicity score formed of worst acute GU + GI +Skin toxicities.

Late GU toxicities occurred in 25.7\% $(\mathrm{n}=66)$ and $5.1 \%$ $(\mathrm{n}=13)$ for $\mathrm{G}-2$ and $\mathrm{G}-3$ respectively. The most common late $\mathrm{G} 2 \mathrm{GU}$ toxicities were irritative symptoms $(\mathrm{n}=26$; $39.4 \%)$, hematuria $(n=23 ; 34.8 \%)$ and urethral stricture $(\mathrm{n}=19 ; 28.8 \%)$; with another six G-3 cases requiring hospitalization and intervention for hematuria and/or stricture. Arm-A had significantly less G-2 (18.1\% vs. $28.6)$ and G-3 (1.4\% vs. $6.5 \%)$ late GU toxicity than Arm-B $(\mathrm{P}=0.008)$ as portrayed in Figure.2. Erectile dysfunction of $\mathrm{G} 2$ or more was encountered in 50 patients in the whole cohort $(19.5 \%)$, albeit rates did not differ between the study arms $(\mathrm{P}=0.39)$.

Regarding late GI side-effects; G-2 and G-3 were observed in $19(7.4 \%)$ and three $(1.2 \%)$ subjects respectively. The prevalent late G-2 GI toxicity was RT proctitis presenting with frequent rectal bleeding in 10 cases $(\mathrm{G} 2 / 3)$ of whom 2 underwent endoscopic laser ablation (G-3). Nonetheless, late GI side-effects were similar between prospective vs. retrospective arms $(8.4 \%$ vs. 8.6; $\mathrm{P}=0.10)$.

Over and above, worst grade late RT-related toxicity and the total late toxicity score (worst GU + GI + erectile dysfunction) were both worse in Arm-B ( $\mathrm{P}=0.008$ and $\mathrm{P}=0.056$; respectively). On MVA, lack of daily CBCT in Arm-B was independently associated with worst late GU toxicity $(\mathrm{P}=0.036)$ after adjusting for $\mathrm{RT}$ total dose and pelvic LN irradiation, in addition to CCI group $(\mathrm{P}=0.04)$. Also study arm was prognostic for higher total late toxicity score $(\mathrm{P}=0.002)$ after adjusting for other factors as shown in Table.3. Meanwhile, African-American race, RT total dose were detrimental for both worst grade late toxicity and total late toxicity score, whereas, higher CCI score was prognostic for higher late toxicity score $(\mathrm{P}<0.05$ for all). Increased RT dose has a protective effect as higher doses were delivered by IGRT using VMAT.

\section{Discussion}

In our study we were able to highlight safe delivery of an escalated curative dose to a homogenous group of intermediate and high-risk localized prostate cancer using IMRT with daily IGRT via CBCT with much improved late toxicity profile and equivalent acute side- 
DOI:10.31557/APJCP.2021.22.1.61

Improved Toxicity with IGRT for Prostate Cancer

Table 2. Radiotherapy and Androgen-Deprivation Therapy Details for Prostate-Cancer Patients Treated with IMRT with (Arm-A) or without Daily Image-Guidance (Arm-B)

\begin{tabular}{|c|c|c|c|}
\hline Characteristic & Arm-A $(\mathrm{N}=72)$ & Arm-B $(\mathrm{N}=185)$ & P-value \\
\hline Median total radiotherapy dose (Gy) & $79.2(78.0-80.0)$ & $74.0(70.0-78.0)$ & $<0.001$ \\
\hline Dose per fraction (Gy) & & & $<0.001$ \\
\hline 1.8 & $53(73.6 \%)$ & $25(13.5 \%)$ & \\
\hline 2 & $19(26.4 \%)$ & $160(86.5 \%)$ & \\
\hline Pelvic lymph-node irradiation & $13(18.1 \%)$ & $1(0.5 \%)$ & $<0.001$ \\
\hline Rectum dose/volume within constraints & & & 0.08 \\
\hline No & $3(4.3 \%)$ & $9(4.9 \%)$ & \\
\hline Borderline & $17(24.3 \%)$ & $13(7 \%)$ & \\
\hline Bladder dose/volume within constraints & & & 0.067 \\
\hline No & $9(12.9 \%)$ & $4(2.2 \%)$ & \\
\hline Borderline & $4(5.7 \%)$ & $27(14.6 \%)$ & \\
\hline Mean PTV maximum dose (\%) & $105.4 \pm 3.1$ & $104.5 \pm 2.7$ & 0.12 \\
\hline ADT administration & $37(51.4 \%)$ & $83(44.9 \%)$ & 0.35 \\
\hline Mean ADT total duration (months) & $12.1 \pm 7.2$ & $11.1 \pm 9.1$ & 0.58 \\
\hline Mean months between ADT and RT & $2.6 \pm 4.2$ & $3.5 \pm 2.4$ & 0.15 \\
\hline
\end{tabular}

IMRT, Intensity-Modulated Radiation Treatment; PTV, Planning Target Volume; ADT, Androgen Deprivation Therapy; RT, Radiotherapy

effects despite similar dosimetry, when compared to a retrospective similar group who had received IMRT with irregular image guidance. This effect was further proven in MVA after adjusting for other factors.

Our findings are in accordance with Zelefsky et al., (2012) who compared IMRT with or without daily position correction with $\mathrm{kV}$ imaging for fiducials and reported more intense late GU toxicities with similar other early and late toxicities. Rates of G2+ late GU side effects in our work were $19.5 \%$ vs. $35.1 \%(\mathrm{P}=0.008)$, for IGRT vs. no IGRT; were less than those in Zelefsky et al., (2008)'s study $(10.4 \%$ vs $20 \%$; $\mathrm{P}=0.02)$, albeit a much higher dose was delivered (86.4 Gy). Lack of daily IGRT and IPSS predicted worse late GU toxicity in MVA similar to our work, nonetheless IPSS was not consistently reported for all candidates $(20 \%)$ of our study and could not be incorporated to the current MVA. Chung et al., (2008) showed that daily $\mathrm{kV}$ imaging of fiducial markers was associated with lower acute GU and GI toxicity in contrast to our work. Nevertheless, all patients of this trial received pelvic RT with a prostatic boost and PTV margins were lower in the IGRT arm ( $3 \mathrm{~mm}$ vs. $1 \mathrm{~cm}$ circumferentially), unlike the current study in which PTV margin was same in both arms ( $1 \mathrm{~cm}$ all around and 0.5 posteriorly). It is noteworthy to mention that having equivalent acute toxicity within our study arms implies improved acute toxicity bearing in mind the significantly higher total dose in the daily CBCT arm (79.2 vs. 74 Gy; $\mathrm{P}<0.001)$. Like ours, Becker-Schiebe et al., (2016) compared higher to a lower dose (77.4 Gy vs. 72 Gy) with or without IGRT. They depicted significantly better acute and similar late RT-related toxicities albeit higher total dose. However, only $16 \%$ received IMRT in contrast to the current work with IMRT in the whole cohort.

Taking in consideration the extra cost for daily IGRT, two European randomized trials compared daily versus weekly IGRT for setup correction (de Crevoisier et al., 2018; Tondel et al., 2018). This extra cost for daily image online guidance includes the price of technology acquisition, more staff work time for each case, more quality assurance and maintenance time, and also less number of patients per machine due to increased treatment time (Perrier et al., 2013). In the French trial which randomized 470 patients to weekly versus daily IGRT using CBCT (77\%), fiducials (EPID or $\mathrm{kV}$ ) or US to deliver a median of $78 \mathrm{~Gy}$, only acute and late GI toxicities

Table 3. Multivariable Cox Regression Analysis Models for Predictors of Late Radiotherapy Induced Toxicity for the Whole Study Cohort [n=257]

\begin{tabular}{|c|c|c|c|c|c|c|c|}
\hline \multirow{2}{*}{$\begin{array}{l}\text { Variable } \\
----\end{array}$} & \multirow{2}{*}{$\begin{array}{l}\text { Response } \\
----\end{array}$} & \multicolumn{2}{|c|}{ Worst late GU toxicity } & \multicolumn{2}{|c|}{ Worst grade late toxicity ${ }^{a}$} & \multicolumn{2}{|c|}{ High total late toxicity score } \\
\hline & & OR $(95 \% \mathrm{CI})$ & P-value & OR $(95 \% \mathrm{CI})$ & $\mathrm{P}$-value & OR $(95 \% \mathrm{CI})$ & P-value \\
\hline Study arm & Arm-B vs. Arm-A & $0.42(0.18-0.92)$ & 0.036 & $1.65(0.54-5.22)$ & 0.384 & $0.67(0.52-0.87)$ & 0.002 \\
\hline Race & Caucasian vs AA & ------- & ------ & $1.87(1.06-3.33)$ & 0.033 & $1.78(1.07-3.08)$ & 0.031 \\
\hline $\begin{array}{l}\text { RT total } \\
\text { dose }^{c}\end{array}$ & Continuous & $0.89(0.77-1.03)$ & 0.13 & $0.84(0.71-0.98)$ & 0.029 & $0.52(0.38-0.71)$ & $<0.001$ \\
\hline \multirow{2}{*}{$\begin{array}{l}\text { CCI group } \\
\text { at diagnosis }\end{array}$} & CCI-0 vs. CCI- 1 & $1.82(0.82-4.05)$ & 0.14 & $1.26(0.56-2.84)$ & 0.581 & $1.97(1.00-3.95)$ & 0.051 \\
\hline & CCI-0 vs. CCI-2+ & $1.98(1.03-3.93)$ & 0.044 & $1.59(0.70-3.62)$ & 0.267 & $1.69(0.88-3.35)$ & 0.121 \\
\hline
\end{tabular}

GU, Genitourinary; OR, Odds Ratio; CI, Confidence Interval; AA, African-American; RT, Radiotherapy; CCI, Charlson Comorbidity Index; a, Adjusted for NCCN risk category and Pelvic lymph-node irradiation; ${ }^{\text {b }}$, Adjusted for clinical T-stage, Pelvic lymph-node irradiation, PTV-maximum dose. 
were improved $(\mathrm{P}=0.027)$, whereas GU toxicities were non-different. Nonetheless, the French control arm had same total dose (78Gy) and IMRT with step-and-shoot technique was utilized in 2 thirds of the cases with $30 \%$ receiving 3D-CRT, unlike ours with VMAT for IMRT delivery in all IGRT cases and with a different median dose of 79.2 vs. 74 Gy in the control group. Also our work included LN irradiation in $18 \%$ of the IGRT group with potentially more GI toxicities vs only one case $(0.5 \%)$ in the other arm, in contrast to prostate only RT in the French trial (de Crevoisier et al., 2018). Furthermore, the other randomized phase 3 study (RIC-trial) randomized 257 patients to receive 78 Gy with either daily CBCT or weekly orthogonal portal images. Like our work, no differences were portrayed for acute toxicities even though PTV margins were much less in the daily CBCT arm ( $7 \mathrm{~mm}$ all around vs $15 \mathrm{~mm})$. It is important to note that the RIC-trial toxicities were derived using patient reported outcomes after RT in comparison to baseline symptoms rather than physician assessed side effects used in this work. Higher acute hematuria $(\mathrm{P}=0.02)$ and nocturia $(\mathrm{P}=0.04)$ with weekly IGRT did not reach the pre-specified significance level of $\mathrm{P}=0.01$ (Tondel et al., 2018). Zhong et al., (2014) compared IGRT using CBCT (2-3 times/week) with a retrospective arm treated using IMRT without IGRT and yielded similar acute and late side effects for both arms. This reinforces that our control arm which included infrequent IGRT (weekly or every 2-3 days) is not better than IMRT without IGRT.

Another aspect in this study is that all IGRT cases received IMRT exclusively via VMAT with 2 arcs for most of the treatment time unlike the control arm that received step-and-shoot technique. In fact, this difference may have an influence on our outcomes. A dosimetric Korean study compared both modalities used in our study as well as Tomotherapy and Proton. Authors concluded that better PTV coverage and superior rectal and bowel sparing in high dose volumes were attained with VMAT compared to other modalities albeit all of them achieved the desired constraints (Lee et al., 2015). Nevertheless, no dosimetric advantage was reported in this work. Poon et al., (2013), compared VMAT (2 arcs) vs. step-and-shoot and results depicted that VMAT is delivered in a significantly shorter time and less monitor units by $61 \%$ and $48 \%$ respectively in addition to better PTV homogeneity index with similar rectal and bladder dosimetry. Another study portrayed mean delivery time of 2 Gy using VMAT (2 arcs) within 2.78 minutes compared to 4.8 minutes for step-and shoot (7-fileds) (Sze et al., 2012). Hence, shorter beam time in VMAT (2 minutes), vs. Step-and-Shoot (5.5 minutes) may have contributed to our findings owing to potentially less intrafractional movement with the shorter VMAT.

Daily image guidance with potential correction of position according to the visualization of PTV soft tissue will take care of setup error and inter-fraction displacement. However, intra-fractional motion still needs to be addressed. Mayyas et al., (2014) compared four different modalities for IGRT (CBCT, US, kV planner images and electromagnetic transponder, Calypso) for daily localization in a group of 27 patients. Intra-fractional motion was assessed using Calypso in 19 cases. The study proposed a minimum margin of $6.6 \mathrm{~mm}$ (anterior/ posterior), $6.8 \mathrm{~mm}$ (superior/inferior) and $3.9 \mathrm{~mm}$ (left /right) to compensate for inter-fraction error ( $4 \mathrm{~mm}$ ) as well as intrafracion motion if IGRT is used with no preferred modality. Thus, image guidance coupled with VMAT with short RT delivery time will deem the intrafractional prostatic motion, changes in bladder filling and rectal surface to be with better control.

Other than the established role of daily IGRT, late RT induced toxicities were independently influenced by African-American race as well as by increased comorbidity assessed by CCI per our exploratory MVA model. Higher baseline CCI score of $2+$ prevailed in almost half of our total cohort emphasizing the extra attention needed to manage RT side-effects in these patients. Hamstra et al., (2013) showed that late RT toxicity was significantly correlated with comorbidity. Nevertheless, only $27 \%$ received IMRT vs 100\% in ours. Furthermore, a Canadian population-based cohort demonstrated an independent role of comorbidity in the development of late side-effects indicating hospital admissions, urological or anorectal procedures in accordance with our findings (Nam et al., 2014).

While we present one of the largest cohorts for a single institution with only intermediate and high risk PCa treated with IMRT, some limitations of this study should be listed. First, comparing with a retrospective group is associated with selection and reporting bias. Second, the prospective arm had a relatively short follow-up with possible more intense late RT-related toxicities down the road. Also, we might have seen more dramatic difference between the study arms if the same RT total dose was utilized which was not possible taking into consideration the established role for dose-escalation nowadays with recommended doses of around $80 \mathrm{~Gy}$; which was not the case between 2005-2007. Again, this was due to the establishment of daily IGRT 2008 onwards in our institute, that prevented a more recent comparative arm. Albeit, we had more detailed treatment and toxicity data much better than commonly used databases like SEER and the NCDB. Finally, we relied only on physicians' notes for grading of RT-induced adverse events with lack of patient filled quality of forms which were not available for the entire cohort. These forms such as Expanded Prostate Index Composite questionnaire (EPIC) reflect the patient's tolerability, bother level and impact of toxicity on daily functioning and are being increasingly adopted and incorporated within endpoints of many published prospective randomized phase 3 trials (Wortel et al., 2016; Brunner et al., 2019).

In conclusion, for patients with localized prostate cancer of intermediate or high-risk, our study suggests that daily image guidance needs to be an essential component in the process of IMRT delivery of contemporary escalated RT doses due to significantly improved late toxicity profile especially for the late genitourinary side-effects with no impact on acute toxicity. African-Americans and those with higher baseline comorbidities should receive more meticulous care during the radiotherapy course and all along follow up as they have relatively higher tendency to develop significant late toxicities. 


\section{Acknowledgements}

\section{Conflict of interest statement}

All authors declare no conflicts of interest.

\section{References}

Becker-Schiebe M, Abaci A, Ahmad T, et al (2016). Reducing radiation associated toxicity using online image guidance (IGRT) in prostate cancer patients undergoing dose-escalated radiation therapy. Rep Pract Oncol Radiother, 21, 188-94.

Bentzen SM, Constine LS, Deasy JO, et al (2010). Quantitative Analyses of Normal Tissue Effects in the Clinic (QUANTEC): An Introduction to the Scientific Issues. Int J Radiat Oncol Biol Phys, 76, S3-9

Bruner DW, Pugh SL, Lee WR, et al (2019). Quality of life in patients with low-risk prostate cancer treated with hypofractionated vs conventional radiotherapy: a phase 3 randomized clinical trial. JAMA Oncol, 1, 664-70

Buglione M, Noale M, Bruni A, et al (2019). Treatment paths for localised prostate cancer in Italy: The results of a multidisciplinary, observational, prospective study (Pros-IT CNR). PLoS One, 14, e0224151

Charlson ME, Pompei P, Ales KL, et al (1987). A new method of classifying prognostic comorbidity in longitudinal studies: Development and validation. J Chronic Dis, 40, 373-83

Chung HT, Xia P, Chan LW, et al (2009). Does image-guided radiotherapy improve toxicity profile in whole pelvic-treated high-risk prostate cancer? Comparison between IG-IMRT and IMRT. Int J Radiat Oncol Biol Phys,73, 53-60.

Common terminology criteria for adverse events: (CTCAE) (2010). National Cancer Institute (U.S.) [Online]. Available: https://evs.nci.nih.gov/ftp1/CTCAE/CTCAE 4.03/ CTCAE 4.03 2010-06-14 QuickReference 5x7.pdf [Accessed November 26 2019].

Cox JD, Stetz J, Pajak TF (1995). Toxicity criteria of the radiation therapy oncology group (RTOG) and the European organization for research and treatment of cancer (EORTC). Int J Radiat Oncol Biol Phys, 31, 1341-6.

Daskivich TJ, Fan KH, Koyama T, et al (2013). Effect of age, tumor risk, and comorbidity on competing risks for survival in a U.S. population-based cohort of men with prostate cancer. Ann Intern Med, 158, 709-17.

de Crevoisier R, Bayar MA, Pommier P, et al (2018). Daily versus weekly prostate cancer image-guided radiotherapy: Phase 3 multicenter randomized trial. Int J Radiat Oncol Biol Phys, 102, 1420-9.

Hammoud R, Patel SH, Pradhan D, et al (2008). Examining margin reduction and its impact on dose distribution for prostate cancer patients undergoing daily cone-beam computed tomography. Int J Radiat Oncol Biol Phys, 71, 265-73

Hamstra DA, Stenmark MH, Ritter T, et al (2013). Age and comorbid illness are associated with late rectal toxicity following dose-escalated radiation therapy for prostate cancer. Int J Radiat Oncol Biol Phys, 85, 1246-53.

Heemsbergen WD, Al-Mamgani A, Slot A, et al (2014). Long-term results of the Dutch Randomized Prostate Cancer Trial: impact of dose-escalation on local, biochemical, clinical failure, and survival. Radiother Oncol, 110, 104-9

Hu JC, Nguyen P, Mao J, et al (2017). Increase in prostate cancer distant metastases at diagnosis in the United States. JAMA Oncol, 3, 705-7.

Kuban DA, Tucker SL, Dong L, et al (2008). Long-term results of the M. D. Anderson randomized dose-escalation trial for prostate cancer. Int J Radiat Oncol Biol Phys, 70, 67-74.

\section{Improved Toxicity with IGRT for Prostate Cancer}

Lee S, Jie Cao Y, Hwan Chang K, et al (2015). Treatment plan comparison of linac step and shoot, tomotherapy, rapidarc, and proton therapy for prostate cancer by using the dosimetrical and the biological indices. J Korean Phys Soc, 67, 7-16.

Mayyas E, Chetty IJ, Chetvertkov M, et al (2013). Evaluation of multiple image-based modalities for image-guided radiation therapy (IGRT) of prostate carcinoma: a prospective study. Med Phys, 40, 041707.

Michalski JM, Yan Y, Watkins-Bruner D, et al (2013). Preliminary toxicity analysis of 3-dimensional conformal radiation therapy versus intensity modulated radiation therapy on the high-dose arm of the Radiation Therapy Oncology Group 0126 prostate cancer trial. Int J Radiat Oncol Biol Phys, 87, 932-8

Moyer VA (2012). US Preventive Services Task Force. Screening for prostate cancer: US Preventive Services Task Force recommendation statement. Ann Intern Med, 157, 120-34.

Nam RK, Cheung P, Herschorn S, et al (2014). Incidence of complications other than urinary incontinence or erectile dysfunction after radical prostatectomy or radiotherapy for prostate cancer: a population-based cohort study. Lancet Oncol, 15, 223-31.

National Comprehensive Cancer Network. NCCN Clinical Practice Guidelines in oncology: prostate cancer Version 4.2019 [Online]. Available: https://www.nccn.org/ professionals/physician_gls/pdf/prostate.pdf [Accessed November 10, 2019]

Perrier L, Morelle M, Pommier P, et al (2013). Cost of prostate image-guided radiation therapy: Results of a randomized trial. Radiother Oncol, 106, 50-8

Poon DM, Chan CK, Chan TW, et al (2018). Consensus statements on the management of metastatic prostate cancer from the Hong Kong Urological Association and Hong Kong Society of Uro-Oncology. BJU Int, 121, 703-15.

Scarbrough TJ, Golden NM, Ting JY, et al (2006). Comparison of ultrasound and implanted seed marker prostate localization methods: implications for image-guided radiotherapy. Int $J$ Radiat Oncol Biol Phys, 65, 378-87.

Siegel RL, Miller KD, Fuchs HE, Jemal A (2021). Cancer statistics, 2021. CA Cancer J Clin, 71, 7-31.

Sze HC, Lee MC, Hung WM, et al (2012). RapidArc radiotherapy planning for prostate cancer: single-arc and double-arc techniques vs. intensity-modulated radiotherapy. Med Dosim, 37, 87-91.

Tondel H, Lund JA, Lydersen S, et al (2018). Radiotherapy for prostate cancer: Does daily image guidance with tighter margins improve patient reported outcomes compared to weekly orthogonal verified irradiation? Results from a randomized controlled trial. Radiother Oncol, 126, 229-35.

Willoughby TR, Kupelian PA, Pouliot J, et al (2006).Target localization and real-time tracking using the calypso 4D localization system in patients with localized prostate cancer. Int J Radiat Oncol Biol Phys, 65, 528-34.

Wortel RC, Incrocci L, Pos FJ, et al (2016). Late side effects after image guided intensity modulated radiation therapy compared to 3D-conformal radiation therapy for prostate cancer: Results from 2 prospective cohorts. Int $J$ Radiat Oncol Biol Phys, 95, 680-9.

Zaorsky NG, Harrison AS, Trabulsi EJ, et al (2013). Evolution of advanced technologies in prostate cancer radiotherapy. Nat Rev Urol, 10, 565-79.

Zaorsky NG, Showalter TN, Ezzell GA, et al (2017). ACR Appropriateness Criteria for external beam radiation therapy treatment planning for clinically localized prostate cancer, part II of II. Adv Radiat Oncol, 2, 437-54.

Zelefsky MJ, Levin EJ, Hunt M, et al (2008). Incidence of late 
rectal and urinary toxicities after three-dimensional conformal radiotherapy and intensity-modulated radiotherapy for localized prostate cancer. Int J Radiat Oncol Biol Phys, 70, 1124-29.

Zelefsky MJ, Kollmeier M, Cox B, et al (2012). Improved clinical outcomes with high-dose image guided radiotherapy compared with non-IGRT for the treatment of clinically localized prostate cancer. Int J Radiat Oncol Biol Phys, 84, 125-9.

Zhong Q, Gao H, Li G, et al (2014). Significance of image guidance to clinical outcomes for localized prostate cancer. Bio Med Res Int, 2014, 860639.

\section{(ब) $(\mathbb{\theta}$}

This work is licensed under a Creative Commons AttributionNon Commercial 4.0 International License. 